\title{
SELETIVIDADE DE HERBICIDAS APLICADOS EM PRÉ E PÓS-EMERGÊNCIA DO ADUBO VERDE PERENE Arachis pintoi ${ }^{1}$
}

\author{
Francisco José Severino ${ }^{2}$ e Pedro J. Christoffoleti ${ }^{3}$
}

\author{
'Parte da dissertação de mestrado em Agronomia, área de concentração Fitotecnia, USP/ESALQ, do primeiro autor. \\ ${ }^{2} E_{n}{ }^{\circ}$. Agro., M.Sc. Universidade de São Paulo, Escola Superior de Agricultura Luiz de Queiroz, Área de Concentração Fitotecnia. Av. \\ Pádua Dias, 11. Caixa Postal 09. Piracicaba, SP 13418-900 \\ ${ }^{3} E_{n}{ }^{\circ}$. Agr ${ }^{\circ}$, PhD., Professor Associado. Universidade de São Paulo, Escola Superior de Agricultura "Luiz de Queiroz", Departamento de \\ Produção Vegetal. pichrist $@$,carpa.ciagri.usp.br
}

\section{RESUMO}

Com o objetivo de avaliar a produção de biomassa seca e toxicidade de herbicidas ao adubo verde perene amendoim forrageiro (Arachis pintoi), quando submetido a pulverizações de herbicidas aplicados em pré e pós-emergência, foram instalados dois experimentos, em casa de vegetação, na Escola Superior de Agricultura "Luiz de Queiroz", Universidade de São Paulo, Departamento de Produção Vegetal, Piracicaba, SP. Os experimentos foram conduzidos em blocos casualizados com quatro repetições. Os herbicidas aplicados em pré-emergência foram: trifluralin $(1,35 \mathrm{~kg} / \mathrm{ha})$, diuron (1,5 kg/ha), oxyfluorfen $(0,6 \mathrm{~kg} / \mathrm{ha})$, atrazine $(2,0 \mathrm{~kg} / \mathrm{ha})$ e metolachlor $(2,88 \mathrm{~kg} / \mathrm{ha})$. Os herbicidas aplicados em pós-emergência foram: fluazifop-p-butil $(0,25 \mathrm{~kg} / \mathrm{ha})$, MSMA $(1,92 \mathrm{~kg} / \mathrm{ha})$, glyphosate $(0,72 \mathrm{~kg} / \mathrm{ha})$, glufosinato de amônio $(0,4 \mathrm{~kg} / \mathrm{ha})$ e sulfosate $(0,96 \mathrm{~kg} / \mathrm{ha})$. As avaliações foram realizadas aos 30, 45, 60 e 75 dias após a aplicação, para os herbicidas em pré-emergência, e aos 7, 14, 21 e 28 dias após a aplicação em pós-emergência. Os resultados permitiram concluir que os herbicidas atrazine, glyphosate, glufosinato de amônio e sulfosate, nas doses aplicadas, não devem ser recomendados, enquanto que os herbicidas trifluralin, oxyflurfen e metolachlor podem ser recomendados com restrições e os herbicidas diuron, fluazifop-p-butil e MSMA podem ser indicados nas doses estudadas. Conclui-se ainda que os herbicidas aplicados em pré-emergência, de forma geral, causaram menor toxicidade e, conseqüentemente, propiciaram menor redução na produção de biomassa seca do Arachis pintoi, do que os herbicidas aplicados em pós-emergência.

Palavras chave: toxicidade, biomassa, amendoim-forrageiro.

\section{ABSTRACT \\ Selectivity of herbicides applied in pre and postemergence of perennial peanut (Arachis pintoi)}

Two experiments were installed under greenhouse conditions at Escola Superior de Agricultura "Luiz de Queiroz", Piracicaba - SP, Brazil, in order to evaluate the dry biomass production and toxicity due to pre and postemergence herbicides on the perennial cover crop Arachis pintoi. The experiment was conducted in randomized complete blocks with four replicates. The herbicides sprayed in preemergence conditions (one experiment) were trifluralin at $0.25 \mathrm{~kg} / \mathrm{ha}$, diuron at $1.5 \mathrm{~kg}$ / ha, oxyfluorfen at $0.6 \mathrm{~kg} / \mathrm{ha}$, atrazine at $2.0 \mathrm{~kg} / \mathrm{ha}$, metolachlor at $2.88 \mathrm{~kg} / \mathrm{ha}$, and a control plot without herbicide applica- 
tion. In the experiment with postemergence herbicides, the treatments were fluazifop-p-butyl at $0.25 \mathrm{~kg} / \mathrm{ha}$, MSMA at 1.92 $\mathrm{kg} / \mathrm{ha}$, glyphosate at $0.72 \mathrm{~kg} / \mathrm{ha}$, ammonium glufosinate at $0.40 \mathrm{~kg} / \mathrm{ha}$, sulfosate at $0.96 \mathrm{~kg} / \mathrm{ha}$ and a control plot without herbicide application. The evaluations were carried out at 30, 45, 60 and 75 days after pre-emergence herbicide treatments, and 7, 14, 21 and 28 days after post-emergence herbicide treatments. The results showed that atrazine, glyphosate, ammonium-glufosinate and sulfosate, at the sprayed rates, should not be applied on the cover crop; however, the herbicides trifluralin, oxyfluorfen and metolachlor can be sprayed at recommended crop rates, with some effects on the development of $A$. pintoi. Diuron, fluazifop-p-butyl and MSMA are fully selective to the green manure. It can also be concluded from the experiment, that, in general, pre-emergence treatments are more selective to $A$. pintoi than post-emergence herbicides, causing lower toxicity and dry biomass reduction, when compared to post-emergence herbicides.

Key words: toxicity, biomass.

\section{INTRODUÇ̃̃O}

O amendoim forrageiro (Arachis pintoi), planta nativa do Brasil Central, cuja propagação ocorre através de sementes e estolhos, vem apresentando bons resultados como cobertura e adubação verde do solo, em pomares de cítricos do estado da Flórida (Estados Unidos da América do Norte) e da Costa Rica. Dentre as características vantajosas de sua utilização destaca-se a capacidade de fixar nitrogênio no solo, controle de erosão, redução de infestação de plantas daninhas, patógenos, insetos e nematóides, não interferindo nas práticas culturais adotadas por estes citricultores (Coleman, 1995).

Numerosos trabalhos de pesquisa relatam a supressão de plantas daninhas causada pelas coberturas verdes, demonstrando o efeito destas no controle de plantas daninhas em condições de campo, sem o uso de herbicidas ou outras formas de manejo (Hartwig, 1989; Enache \& Ilnick, 1990; Facelli \& Pickett, 1991; White \& Scott, 1991; DeHaan et al., 1994).

Estudos da supressão de plantas daninhas pelas coberturas verdes, na cultura da ervilha, demonstraram bons resultados, variando entre os diferentes tipos de materiais utilizados (Al-Khatib et al., 1997). Pizarro et al. (1997), estudaram a produção e persistência de Arachis pinto $i$ associada com Paspalum maritimum, em pastagens do Cerrado brasileiro, sem o uso de herbicidas para o controle de plantas infestantes e verificaram que, ao final de dois anos, Arachis pintoi representava $60 \%$ da cobertura vegetal do solo, apresentando boas condições sanitárias e de vigor. Os autores também notaram resultados positivos no controle de erosão e plantas daninhas. Por outro lado, outros autores mencionam que a infestação de plantas daninhas constituiu-se na principal limitação no estabelecimento de algumas espécies de adubos verdes em culturas anuais, reduzindo, em alguns casos, a produtividade econômica destas (Mohler, 1991).

Desta maneira, embora a adubação verde, utilizada como cobertura viva, possa fazer parte de um conjunto de medidas efetivas para a redução da infestação de populações de plantas daninhas, existe a necessidade de se realizar um manejo de plantas daninhas adequado para estas espécies, até que estejam estabelecidas, com o objetivo de prevenir a invasão e o estabelecimento de plantas infestantes (Teasdale, 1998).
Entre as medidas utilizadas para o controle de plantas daninhas, bem como regular o crescimento de adubos verdes, utilizados como cobertura viva do solo, está o uso de herbicidas. O herbicida é utilizado com dois objetivos: eliminar a competição da planta daninha durante o estabelecimento do adubo verde e reduzir o crescimento dos adubos verdes que eventualmente possam competir com a cultura em convivência.

A alfafa (Medicago sativa) pode ter seu crescimento suprimido por doses subletais de atrazine, mas, no entanto, pode regenerar-se a partir de brotações das gemas dos ramos inferiores, revertendo desta forma o efeito do herbicida (Eberlein et al., 1992). Outros autores também verificaram que subdoses de herbicidas podem ser empregadas para proporcionar o controle do crescimento de coberturas vivas (Elkins et al., 1983; Hartwig, 1987).

Desta forma, este experimento estudou os efeitos da aplicação de herbicidas em pré e pós-emergência, através da avaliação de toxicidade e de produção de biomassa seca do Arachis pintoi, visando obter informações técnicas que possam contribuir no controle do crescimento desse adubo verde e controle de plantas daninhas.

\section{MATERIAL E MÉTODOS}

O experimento foi conduzido em casa-de-vegetação do Departamento de Produção Vegetal da Escola Superior de Agricultura "Luiz de Queiroz", campus da ESALQ/USP, em Piracicaba, SP, Brasil.

$\mathrm{O}$ ensaio constou de duas fases. Na primeira fase foi coletado solo, durante a primeira semana de setembro de 1999 . de uma área ocupada por um pomar de abacate (Persea americana), em solo argiloso, com $38 \%$ de argila, $19 \%$ de silte e $43 \%$ de areia, pH 5,3 e $37 \mathrm{~g} / \mathrm{dm}^{3}$ de matéria orgânica. Essa área exibia alta infestação natural de plantas daninhas, composta por mono e dicotiledôneas. A coleta do solo foi feita com trado de $4,3 \mathrm{~cm}$ de diâmetro, a $10 \mathrm{~cm}$ de profundidade e de maneira aleatória (o volume de solo coletado foi o necessário para encher os vasos). A seguir o solo foi colocado em sacos plásticos, levado ao laboratório, peneirado (peneira com malha de $0,280 \mathrm{~mm}$ ) para desfazer possíveis agregados e colocado em vasos plásticos de $205 \mathrm{~cm}^{3}$ de capacidade. Em se- 
guida, foram cuidadosamente semeadas 10 sementes de Arachis pintoi/vaso, inclusive nos vasos onde seriam, posteriormente, realizados os tratamentos em pós-emergência. Antes da aplicação em pré-emergência os vasos foram irrigados com o equivalente a uma chuva de $15 \mathrm{~mm}$, visando melhorar as condições de umidade. A seguir foram submetidos à aplicação de herbicidas, em pré-emergência tanto do Arachis pintoi como das plantas daninhas. As pulverizações foram realizadas em câmara de aplicação, com pressão de $2,8 \mathrm{~kg} / \mathrm{cm}^{2}$, utilizando-se pontas $110.02 \mathrm{E}$, com volume de calda aplicado equivalente a $300 \mathrm{l} / \mathrm{ha}$. Os herbicidas utilizados estão descritos na Tabela 1. Em seguida, todos os vasos foram levados para a casa de vegetação, onde foram submetidos a sistema de regas diárias. O sistema de irrigação era composto por microaspersores controlados, que irrigavam aproximadamente $5 \mathrm{~mm}$ cada vez que eram acionados. A casa de vegetação possuia um sistema automático de controle de temperatura e umidade relativa, através de ventiladores, e a luminosidade era controlada por uma cobertura de sombrite, que se distendia automaticamente conforme a luminosidade incidente.

Depois de 14 dias da instalação da primeira fase, foi montada a segunda, quando o Arachis pinto $i$ apresentava boas condições vegetativas para que as aplicações em pós-emergência pudessem ser realizadas. Assim, todos os vasos que seriam submetidos à aplicação em pós-emergência foram retirados da casa-de-vegetação e levados para a câmara de apli- cação, onde foram utilizadas as mesmas condições das aplicações em pré-emergência. Os tratamentos em pós-emergência estão representados na Tabela 2 . Neste caso, os vasos só retornaram para a casa de vegetação depois de oito horas após a aplicação, onde foram novamente submetidos a regas freqüentes, de três a quatro vezes por dia, até o final das avaliações.

O delineamento experimental utilizado, em ambos os experimentos, foi o de blocos casualizados com quatro repetições. Durante a condução do experimento, foram avaliadas a toxicidade ao Arachis pintoi para o experimento em préemergência, aos 30, 45, 60 e 75 dias após a aplicação (DAA) e para o experimento em pós-emergência aos 7, 14, 21 e 28 DAA. Estas avaliações de toxicidade foram realizadas por meio da Escala EWRC, onde I significa ausência total de injúria, e 9 morte total da planta. No final foi feita a avaliação da produção de biomassa seca do Arachis pintoi em todos os tratamentos. Na casa de vegetação foi realizado, cuidadosamente, o corte da parte aérea de dez plantas/vaso de Arachis pintoi e essas amostras foram colocadas em pequenos sacos de papel, devidamente identificadas e levadas ao laboratório para secagem em estufa a $50^{\circ} \mathrm{C}$, durante 48 horas, e pesagem em balança de precisão.

Os dados de biomassa seca obtidos foram analisados por meio de análise de variância, com o auxílio do Sistema de Análise Estatística - SANEST, sendo as médias comparadas entre si através do teste Tukey, ao nível de 5\% de proba-

Tabela 1. Herbicidas utilizados no experimento instalado em condições de pré-emergência. Experimento de casa-de-vegetação. USP/ESALQ, Piracicaba, SP. 2000.

\begin{tabular}{|c|c|c|c|c|c|}
\hline \multicolumn{2}{|c|}{ Herbicida } & \multirow{2}{*}{ Formulação } & \multirow{2}{*}{$\begin{array}{c}\text { Concentração } \\
(\mathrm{g} / \mathrm{l})\end{array}$} & \multicolumn{2}{|c|}{ Dose } \\
\hline Nome técnico & Nome Comercial & & & i.a. (kg/ha) & p.c. (1/ha) \\
\hline Trifluralin & Trifl. Nortox & CE & 450 & 1,35 & 3,0 \\
\hline Diuron & Karmex & $\mathrm{SC}$ & 500 & 1,50 & 3,0 \\
\hline Oxyfluorfen & Goal $\mathrm{Br}$ & $\mathrm{CE}$ & 240 & 0,60 & 2,5 \\
\hline Atrazine & Gesaprim & $\mathrm{SC}$ & 500 & 2,00 & 4,0 \\
\hline Metolachlor & Dual & CE & 960 & 2,88 & 3,0 \\
\hline
\end{tabular}

i.a. $=$ ingrediente ativo; p.c. $=$ produto comercial; $\mathrm{CE}=$ Concentrado emulsionável; $\mathrm{SC}=$ Suspensão concentrada.

Tabela 2. Herbicidas utilizados no experimento instalado em condições de pós-emergência. Experimento de casa-de-vegetação. USP/ESALQ, Piracicaba, SP. 2000.

\begin{tabular}{|c|c|c|c|c|c|}
\hline \multicolumn{2}{|c|}{ Herbicida } & \multirow{2}{*}{ Formulação } & \multirow{2}{*}{$\begin{array}{c}\text { Concentração } \\
(\mathrm{g} / \mathrm{l})\end{array}$} & \multicolumn{2}{|c|}{ Dose } \\
\hline Nome técnico & Nome Comercial & & & i.a. (kg/ha) & p.c. $(1 / h a)$ \\
\hline Fluazifop-p-butil & Fusilade & $\mathrm{CE}$ & 125 & 0,25 & 2,0 \\
\hline MSMA & Daconate & SA & 480 & 1,92 & 4,0 \\
\hline Glyphosate & Roundup & SA & 360 & 0,72 & 2,0 \\
\hline Glufosinato de amônio & Finale & $\mathrm{CE}$ & 200 & 0,40 & 2,0 \\
\hline Sulfosate & Zapp & SA & 480 & 0,96 & 2,0 \\
\hline
\end{tabular}

i.a. $=$ ingrediente ativo; p.c. $=$ produto comercial; $\mathrm{CE}=$ Concentrado emulsionável; $\mathrm{AS}=$ Suspensão aquosa. 
bilidade. Os resultados de toxicidade não foram avaliados estatisticamente.

\section{RESULTADOS E DISCUSSÃO}

Nas Tabelas 3 e 4 estão apresentadas as médias de produção de biomassa seca (em g/vaso) e de toxicidade do Arachis pintoi (Escala EWRC), quando submetido à aplicação de herbicidas em pré e pós-emergência. No que se refere à produção de biomassa seca do Arachis pintoi, quando submetido a aplicações em pré-emergência, verifica-se que a média dos tratamentos com o herbicida diuron foi a única que não diferiu significativamente da média da testemunha, enquanto que as médias dos tratamentos com os herbicidas trifluralin, oxyfluorfen e metolachlor não apresentaram diferenças sig- nificativas entre si. No entanto, a média do tratamento com o herbicida atrazine apresentou diferenças significativas quando comparada com as demais médias (Tabela 3).

Com relação à toxicidade do Arachis pintoi, quando submetido a aplicações de herbicidas em pré-emergência, nota-se que, na primeira avaliação, aos $30 \mathrm{DAA}$, os herbicidas trifluralin, diuron e metolachlor obtiveram resultados próximos do resultado da testemunha. O oxyfluorfen apresentou injúria leve, enquanto que atrazine já apresentava grande diferença dos demais tratamentos, causando injúrias severas. Nas avaliações seguintes, aos 45 e 60 DAA, os resultados se mantiveram quase inalterados, sendo que o atrazine já apresentava morte total das plantas. Na última avaliação, realizada aos 75 DAA, os herbicidas trifluralin, diuron, oxyfluorfen e metolachlor não apresentaram nenhum tipo de toxicidade ao Arachis pintoi, quando comparados com a testemunha, enquanto que o atrazine levou a planta à morte.

Tabela 3. Médias de produção de biomassa seca (g/vaso) e de toxicidade do Arachis pintoi submetido a aplicações de herbicidas em pré-emergência. Experimento de casa-de-vegetação. USP/ESALQ, Piracicaba, SP. 2000.

\begin{tabular}{|c|c|c|c|c|c|c|}
\hline \multirow{3}{*}{ Tratamento } & \multirow{3}{*}{$\begin{array}{c}\text { Dose } \\
(\mathrm{kg} / \mathrm{ha})\end{array}$} & \multirow{3}{*}{$\begin{array}{c}\text { Biom assa } \\
\text { Seca }^{1} \\
(\text { g/vaso) }\end{array}$} & \multicolumn{4}{|c|}{ Dias após aplicação dos herbicidas } \\
\hline & & & 30 & 45 & 60 & 75 \\
\hline & & & \multicolumn{4}{|c|}{ Fitotoxicidade } \\
\hline Trifluralin & 1,35 & $5,1 b^{2}$ & 1,0 & 1,2 & 1,2 & 1,0 \\
\hline Diuron & 1,50 & 6,0 a & 1,2 & 1,0 & 1,0 & 1,0 \\
\hline Oxyfluorfen & 0,60 & $5,1 \mathrm{~b}$ & 2,8 & 2,0 & 1,0 & 1,0 \\
\hline Atrazine & 2,00 & $0,0 \mathrm{c}$ & 7,8 & 9,0 & 9,0 & 9,0 \\
\hline Metolachlor & 2,88 & $4,8 \mathrm{~b}$ & 1,2 & 1,2 & 1,0 & 1,0 \\
\hline Testemunha & - & $6,2 \mathrm{a}$ & 1,0 & 1,0 & 1,0 & 1,0 \\
\hline C.V. & - & 8,9 & - & - & - & - \\
\hline D.M.S. & - & 0,8 & - & - & - & - \\
\hline
\end{tabular}

'Para a análise estatística os dados foram transformados em $\sqrt{x+0,5}$.

${ }^{2}$ Médias seguidas por letras distintas na coluna diferem entre si a $5 \%$ de significância pelo teste de Tukey.

Tabela 4. Médias de produção de biomassa seca (g/vaso) e de fitotoxicidade do Arachis pintoi submetido a herbicidas aplicados em pós-emergência. Experimento de casa-de-vegetação. USP/ESALQ, Piracicaba, SP. 2000.

\begin{tabular}{|c|c|c|c|c|c|c|}
\hline \multirow{3}{*}{ Tratamento } & \multirow{3}{*}{$\begin{array}{c}\text { Dose } \\
(\mathrm{kg} / \mathrm{ha})\end{array}$} & \multirow{3}{*}{$\begin{array}{c}\text { Biomassa } \\
\text { Seca }^{1} \\
(\text { g/vaso })\end{array}$} & \multicolumn{4}{|c|}{ Dias após aplicação dos herbicidas } \\
\hline & & & 7 & 14 & 21 & 28 \\
\hline & & & \multicolumn{4}{|c|}{ Fitotoxicidade } \\
\hline Fluazifop-p-butil & 0,25 & $5,3 \mathrm{a}^{2}$ & 1,2 & 1,2 & 1,0 & 1,0 \\
\hline MSMA & 1,92 & $5,8 \mathrm{a}$ & 3,8 & 3,8 & 2,0 & 1,2 \\
\hline Glyphosate & 0,72 & $0,0 \mathrm{~b}$ & 7,2 & 8,8 & 9,0 & 9,0 \\
\hline Glufosin ato & 0,4 & $0,0 \mathrm{~b}$ & 8,0 & 8,2 & 9,0 & 9,0 \\
\hline Sulfosate & 0,96 & $0,0 \mathrm{~b}$ & 7,2 & 8,8 & 9,0 & 9,0 \\
\hline Testemunha & --- & $6,5 \mathrm{a}$ & 1,0 & 1,0 & 1,0 & 1,0 \\
\hline C.V. & - & 22,9 & - & - & - & - \\
\hline D.M.S. & - & 1,3 & - & - & - & - \\
\hline
\end{tabular}

'Para a análise estatística os dados foram transformados em $\sqrt{\mathrm{x}+0,5}$.

${ }^{2}$ Médias seguidas por letras distintas na coluna diferem entre si a 5\% de significância pelo teste de Tukey. 
Com respeito à produção de biomassa seca do Arachis pintoi, quando submetido a aplicações em pós-emergência, nota-se que as médias dos tratamentos com os herbicidas fluazifop-p-butil e MSMA não apresentaram diferenças significativas em comparação com a média da testemunha, enquanto que as médias dos tratamentos com os herbicidas glyphosate, glufosinato de amônio e sulfosate não diferiram significativamente quando comparadas entre si, mas mostraram diferenças significativas em comparação com a média dos tratamentos com fluazifop-p-butil, MSMA e testemunha (Tabela 4).

Com relação à avaliação de toxicidade do Arachis pintoi, quando submetido a aplicações de herbicidas em pósemergência, nota-se que na primeira avaliação, aos 7 DAA, os herbicidas glyphosate, glufosinato de amônio e sulfosate causaram injúrias severas no Arachis pintoi, enquanto que o MSMA apresentou injúria leve e o tratamento com fluazifopp-butil demonstrou ausência de injúria. Nas avaliações subseqüentes, aos 14 e 21 dias após a aplicação, os herbicidas glyphosate, glufosinato de amônio e sulfosate causaram a morte da planta, enquanto que os resultados dos outros dois tratamentos mantiveram-se praticamente constantes. No final da última avaliação, aos 28 dias, os tratamentos fluazifopp-butil e MSMA demonstravam ausência total de injúria, enquanto que os outros três tratamentos causaram a morte da planta.

\section{CONCLUSÕES}

Os herbicidas fluazifop-p-butil $(0,25 \mathrm{~kg} / \mathrm{ha})$ e MSMA $(1,92 \mathrm{~kg} / \mathrm{ha})$ são seletivos ao Arachis pintoi.

Os herbicidas trifluralin, $(1,35 \mathrm{~kg} / \mathrm{ha})$, oxyfluorfen $(0,6$ $\mathrm{kg} / \mathrm{ha})$ e metolachlor $(2,88 \mathrm{~kg} / \mathrm{ha})$ apresentaram boa seletividade, no entanto reduziram a produção de biomassa seca do Arachis pintoi e devem ser recomendados com restrições.

Os herbicidas atrazine $(2,0 \mathrm{~kg} / \mathrm{ha})$, glyphosate $(0,72$ $\mathrm{kg} / \mathrm{ha})$, glufosinato de amônio $(0,4 \mathrm{~kg} / \mathrm{ha})$ e sulfosate $(0,96$ $\mathrm{kg} / \mathrm{ha}$ ) não devem, nestas doses, ser indicados para o controle de plantas daninhas no Arachis pintoi.

\section{LITERATURA CITADA}

AL-KHATIB, K.; LIBBEY, C.; BOYDSTON, R. Weed suppression with Brassica green manure crops in green pea. Weed Science, v.45, p.439-445, 1997.
COLEMAN, R.G. Perennial peanuts - potential looms larger in citrus. Citrus Industry, p.43-44, 1995.

DEHAAN, R.L.; WYSE, D.L.; EHLKE, N.J. Simulation of spring-seeded smother plants for weed control in corn. Weed Science, v.42, p.35-43. 1994.

EBERLEIN. C.V.; SHEAFFER, C.C.; OLIVEIRA, V.F. Corn growth and yield in an alfalfa living mulch system. Agricultural Products Journal, v.5, p.332-339, 1992.

ELKINS, D.; FREDERKING, D.: MARASHI, R. Living mulch for no-tillage corn and soybeans. Journal of Soil and Water Conservation, v.38, p.431-433, 1983.

ENACHE, A.J.; ILNICKI, R.D. Weed control by subterranean clover used as living mulch. Weed Technology, v. 4, p.534-538, 1990.

FACELLI, J.M; PICKETT, S.T.A. Plant litter: its dynamics and effects on plant community structure. Botany Reviews, v.57, p.1-32, 1991.

HARTWIG, N.L. Cropping practices using crownvetch in conservation tillage. In: POWER, J.F. The role of legumes in conservation tillage systems. Ankeny, Iowa: Soil Conservation Society of America, 1987. p. 109-110.

HARTWIG, N.L. Influence of a crownvetch living mulch on dandelion invasion in corn. In: NORTHEAST WEED SCIENCE SOCIETY, Proceedings... v.33, p.25-28, 1989.

MOHLER, C.L. Effects of tillage and mulch on weed biomass and sweet corn yield. Weed Technology, v.5, p.545$552,1991$.

PIZARRO, E.A.; RAMOS, A.K.B.; CARVALHO, M.A. Produccion y persistencia de siete acceiones de Arachis pintoi asociadas con Paspalum maritimum en el cerrado brasileño. Pasturas Tropicales, v.19, n.2, p.4044, 1997.

TEASDALE, J.R. Cover crops, smother plants, and weed management. Arbor Press (Periódico), 1998. p.247270.

WHITE, J.G.; SCOTT, T.W. Effects of perennial forage-legume living mulches on no-till winter wheat and rye. Field Crops Research, v.28, p. 135-148, 1991. 
